\title{
Introduction to Volume 1 Issue 2 of Journal of Cancer Metastasis and Treatment
}

\section{Lucio Miele}

Stanley Scott Cancer Center, Louisiana State Health Sciences Center and Louisiana Cancer Research Consortium, New Orleans, LA 70112, USA.

Correspondence to: Prof. Lucio Miele, Stanley Scott Cancer Center, Louisiana State Health Sciences Center and Louisiana Cancer Research Consortium, New Orleans, LA 70112, USA. E-mail: 1miele@1suhsc.edu

It is my privilege to introduce the second issue of the Journal of Cancer Metastasis and Treatment. In keeping with the format we have chosen for our journal, this issue contains two reviews focusing on fields of great translational and clinical interest, five original articles, and three case reports. The first review discusses the rapidly evolving field of circulating tumor cells (CTC) as cancer biomarkers. Since the introduction of the first Food and Drug Administration approved CTC test to assess the progression of metastatic breast, colorectal and prostate cancer, CTC have generated tremendous interest among clinicians seeking sensitive progression biomarkers and basic scientists interested in isolating and studying these cells. A number of new sorting, capture, and enumeration technologies are being evaluated. Competing technologies have emerged, such as circulating tumor DNA. The review by Potdar and Lotey summarizes the current experience with CTC as diagnostic and prognostic biomarkers and the future of this technology. The review by Alfonso et al. describes recent progress in our understanding of urothelial cancer, a poorly understood malignancy that can be very difficult to treat, based on novel translational science. The original articles cover a variety of translational and clinical topics: the rapidly growing field of extracellular microRNA (miRNA) detection and quantification, as applied to medulloblastoma; the management of diffuse large B-cell non-Hodgkin lymphoma (NHL) in elderly Egyptian patients; the prognostic value of GATA3 and FOXA1 detection in breast cancer; current clinical criteria

\begin{tabular}{|l|l|}
\hline \multicolumn{2}{|c|}{ Access this article online } \\
\hline Quick Response Code: & Website: \\
\hline & www.jcmtjournal.com \\
\cline { 2 - 2 } & \\
\hline
\end{tabular}

for the management of squamous non-small cell lung cancer (NSCLC); and the pharmacological properties of Withania somnifera extract in a triple-negative breast cancer model. miRNAs and other non-coding RNAs in biological fluids promise to revolutionize the world of biomarkers. However, there remain significant technical issues surrounding the reproducible quantification of miRNAs for clinical purposes. The manuscript by Shalaby et al. is an example of progress in this highly promising field. The manuscript by Zeeneldin et al. describes the experience of treating diffuse large B-cell NHL in a geriatric setting where safety and efficacy considerations have be balanced against each other and where monoclonal antibodies are not always available. The study by Chivukula et al. proposes the intriguing hypothesis that immunohistochemical detection of the transcription factor GATA3 and "pioneer" factor FOXA1 has prognostic value in breast cancer. The manuscript by Savini et al. describes the experience of this group treating squamous NSCLC, analyzing variables associated with improved survival in their patient population. The article by Ray et al. describes the effect of a promising natural product, an extract from well-known medicinal plant Withania somnifera, on the production of cytokines associated with metastasis in a standard triple-negative breast cancer model. Finally, the three case reports describe unusual presentations, including histologically different metastases from an unknown primary lesion, orbital metastasis from rectal carcinoma and a mature spinal teratoma presenting in an elderly patient.

I hope you enjoy reading this issue, and the ones that will follow.

How to cite this article: Miele L, Introduction to Volume 1 Issue 2 of Journal of Cancer Metastasis and Treatment. J Cancer Metastasis Treat 2015;1:43.

Received: 11-06-2015; Accepted: 18-06-2015.

Source of Support: Nil, Conflict of Interest: None declared. 